\title{
Formulation Development and Optimization of Phase- Transition W/O Microemulsion In Situ Gelling System for Ocular Delivery of Timolol Maleate in the Treatment of Glaucoma
}

\author{
Prigneshkumar Patel ${ }^{1}$ (D), Gayatri Patel 2,*(D) \\ 1 Manager-1, FR\&D - Formulation Research \& Development (Non -Orals), Sun Pharmaceutical Industries Ltd, Tandalja, \\ Nima Compound, Vadodara, Gujarat, India; prigneshspatel@gmail.com (P.P.); \\ 2 Charotar University of Science and Technology, Ramanbhai Patel College of Pharmacy, Department of Pharmaceutics \& \\ Pharmaceutical Technology, CHARUSAT CampusChanga-388 421, Gujarat, India; gayatripatel26@ gmail.com (G.P.); \\ * Correspondence: gayatripate126@gmail.com;
}

Scopus Author ID 35500684500

Received: 30.07.2020; Revised: 28.08.2020; Accepted: 29.08.2020; Published: 1.09.2020

\begin{abstract}
The present investigation is aimed to prepare and evaluate the micro emulsion-based phase transition ocular system for delivery of Timolol maleate in the treatment of glaucoma. Timolol maleate is used in the first line of treatment in open-angle glaucoma, belonging to BCS class-I having good solubility and permeability. The rapid precorneal elimination of conventional formulation containing class I drugs exhibits poor therapeutic effect and bioavailability. So, microemulsion (ME) based phase transition systems were formulated and characterized. ME based phase transition system was formulated using Ethyl oleate as oil and CremophorEL as a surfactant, Span 20 as Co-surfactant, and Sorbic acid as a preservative. These systems undergo a phase transition from water-in-oil (w/o) ME to liquid crystalline (LC) state and to coarse emulsion (EM) with a change in viscosity depending on dilution with tear fluid \& water content. Prepared microemulsions were characterized for average globule size, zeta potential, $\mathrm{pH}$, conductance, in-vitro gelling capacity. The optimized formulation was selected based on desirable attributes and was further characterized and compared with marketed ophthalmic gel-forming marketed solution of Timolol maleate (TIMOPTIC-XE ${ }^{\circledR}$ ). All the results of the characterization were satisfactory. The optimized water-in-oil (w/o) microemulsion showed droplet size $23.47 \mathrm{~nm}$, the zeta potential of $0.253 \mathrm{mV}, \mathrm{pH}$ of 7.2 , the conductance of $0.25 \mathrm{mS}$, and drug content of 99.64\%. The phase transition w/o ME provides the fluidity for installation with its viscosity being increased due to phase transition after application increasing ocular retention while retaining the therapeutic efficiency. The in- vitro drug release and IOP reduction with optimized formulation were found comparable and less fluctuating compared to marketed formulation. Optimized formulation was found stable during the accelerated stability study. The developed phase transition w/o ME formulation would be able to offer benefits, such as increased residence time, prolonged drug release, reduction in dosing frequency, and thereby it will improve patient compliance.
\end{abstract}

Keywords: Open-angle glaucoma; Phase-transition; Viscosity; Controlled release.

(C) 2020 by the authors. This article is an open-access article distributed under the terms and conditions of the Creative Commons Attribution (CC BY) license (https://creativecommons.org/licenses/by/4.0/).

\section{Introduction}

Ophthalmic drug delivery is challenging due to the structure and physiology of the human eye where corneal epithelium, stroma, and secretion of lachrymal fluid hinder the permeation of drug molecules. The corneal epithelium and stroma are the rate-limiting barriers 
for the hydrophilic and lipophilic drugs, respectively, whereas the lachrymal secretion is responsible for the cleaning of the anterior surface of the eye [1]. For a formulator, it is a significant challenge to overcome these protective barriers without damaging the tissue. The large fraction of ocular drug delivery is in the form of eye drops for topical administration into the lower $c u l$-de-sac. The dosage form properties like hydrogen ion concentration, osmolality, viscosity, and instilled volume affects the pre-corneal retention [2,3]. In order to improve the residence time of drugs in the cul-de-sac, gels and semisolid based preparations were developed, but such systems posed the problems of particulate matter, inconvenience in sterilization, greasiness, blurred vision and hence are not well received $[4,5]$. There is a need to develop a more efficient delivery system that can enhance ocular bioavailability, ocular retention, and absorption of drugs [6].

Recently, microemulsions have emerged as a promising alternative dosage form for ocular drug delivery. Microemulsions (ME) are spontaneously forming isotropic, thermodynamically stable, transparent (or translucent) systems of oil, water, surfactant, and a co-surfactant with dispersed phase usually in the range of 10-100nm. Structurally, MEsare classified as oil-in-water $(\mathrm{o} / \mathrm{w})$, water in oil (w/o), and bi-continuous $[7,8]$. The nanodroplet size provides better membrane adherence and transport of drug molecules. The interface between oil and water is stabilized by an ultra-low interfacial tension created by an appropriate combination of surfactants and/or co-surfactants, which subsequently leads to a simultaneous and spontaneous increase in the interfacial area [9]. The large interfacial area formed may divide itself into a large number of small droplets of either oil in water or water in oil in order to decrease the free energy of the system. The selection of each component, along with their specific concentration ratio is most important for stable ophthalmic ME. The ophthalmic compatibility and purity of excipients need careful consideration for [10].

Several studies suggested the application of phase transition o/w ME with an enhanced drug penetration to the anterior segment for a prolonged period compared with a conventional preparation [11-14]. These systems undergo an in-vivo phase change from ME to liquid crystalline (LC) and to finally coarse emulsion (EM) upon dilution with tear fluid, which subsequently enhances the viscosity [15]. However, there are no studies reported to explore the changes in viscosity during the phase transition to LC phase and optimize it as in situ gelling systems for ophthalmic drug delivery.

The present investigation is aimed to evaluate the ME-based phase transition in situ gelling systems for ophthalmic drug delivery. The phase transitions ME formulations with various combinations of oils, surfactants, and co-surfactants were developed and evaluated with the aim of investigating the influence of phase transition on the release and therapeutic efficacy of the model drug. In this study, the Timolol maleate was used as a model drug, which is mostly used as conventional eye drops with high dosing frequency to treat open-angle glaucoma. There is a need for a better ophthalmic delivery system, which can enhance drug residence time, ocular bioavailability, and eventually decrease the dosing frequency.

\section{Materials and Methods}

\subsection{Materials.}

Timolol maleate was obtained as a gift sample from Centaur Pharmaceutical Ltd, Mumbai. Ethyl oleate was purchased from Indo Amines Ltd. Mumbai. Isopropyl myristate was purchased from S. D. Fine Chemicals Ltd., Mumbai. Tween 80, Tween 20, and Span 20 were 
purchased from Croda, Mumbai. Cremophore EL was purchased from BASF, Germany. Capmul MCM was obtained as a gift sample from IMCD (India) Mumbai. All other chemicals and reagents were used for an analytical grade.

\subsection{Methods.}

\subsubsection{Selection of formulation components.}

The selection of formulation components was carried out by solubility study and drug excipients compatibility study. The solubility study was carried out by taking $10 \mathrm{ml}$ of each selected solvent, i.e., oil, surfactant, and co-surfactant in different beakers. The excess amount of drug Timolol maleate was added and stirred for 48 hours at $30^{\circ} \mathrm{C}$ on a magnetic stirrer followed by centrifugation at 7500RPM for $10 \mathrm{~min}$. The concentration of Timolol maleate in the supernatant was measured by HPLC. Then drug solubility $(\mathrm{mg} / \mathrm{ml})$ was calculated in each selected solvent. The drug excipient compatibility study was conducted by mixing the drug with the same volume of selected components. The mixture was stored at room temperature and at $40^{\circ} \mathrm{C}$ for 1 month and observed for chemical compatibility, including drug assay and physical compatibility, including precipitation, crystallization, phase separation, and color change. The components found physically and chemically compatible with the drug were selected for preliminary formulations $[16,17]$.

\subsubsection{Preparation of preliminary formulations.}

Preliminary compositions of the w/o ME system were prepared by the auto emulsification method for the selection of oil, surfactant, and co-surfactant. The preliminary formulations shown in Table 1 were prepared by taking different ratios of oil, water, surfactant, and co-surfactant. The drug was dissolved in distilled water, followed by the addition of surfactant with slow stirring to avoid foam generation. In another beaker, oil, surfactant, and co-surfactant were mixed similarly. The aqueous phase was slowly added into the oil phase with continuous stirring and stored at room temperature.

Table 1. Composition of preliminary batches for excipient selection.

\begin{tabular}{l|c|c|c|c|c|c|c|c} 
Ingredients & Batch & Batch & Batch & Batch & Batch & Batch & Batch & Batch \\
& A & B & C & D & E & F & G & H \\
\hline Tween 20 & $40 \mathrm{ml}$ & $40 \mathrm{ml}$ & - & - & - & - & - & - \\
\hline Tween 80 & - & - & $40 \mathrm{ml}$ & $40 \mathrm{ml}$ & & $40 \mathrm{ml}$ & $40 \mathrm{ml}$ & \\
\hline Cremophore EL & - & - & - & - & - & - & - & $40 \mathrm{ml}$ \\
\hline Solutol HS 15 & - & - & - & - & $26.86 \mathrm{ml}$ & - & - & - \\
\hline Span 20 & $15 \mathrm{ml}$ & $15 \mathrm{ml}$ & $15 \mathrm{ml}$ & $15 \mathrm{ml}$ & $12.88 \mathrm{ml}$ & $15 \mathrm{ml}$ & $15 \mathrm{ml}$ & $15 \mathrm{ml}$ \\
\hline Ethyl Oleate & - & $40 \mathrm{ml}$ & - & $40 \mathrm{ml}$ & $43.62 \mathrm{ml}$ & $40 \mathrm{ml}$ & - & $40 \mathrm{ml}$ \\
\hline Capmul MCM & $40 \mathrm{ml}$ & - & $40 \mathrm{ml}$ & - & - & - & - & - \\
\hline Isopropyl Myristate & - & - & - & - & - & - & $40 \mathrm{ml}$ & - \\
\hline Distilled water & $5 \mathrm{ml}$ & $5 \mathrm{ml}$ & $5 \mathrm{ml}$ & $5 \mathrm{ml}$ & $16.62 \mathrm{ml}$ & $5 \mathrm{ml}$ & $5 \mathrm{ml}$ & $5 \mathrm{ml}$
\end{tabular}

The w/o ME formulations were diluted gradually by the addition of artificial tear fluid (ATF), and the volume of ATF was measured during phase transition, and the pseudo ternary phase diagram was constructed. During phase transient, various parameters like the transparency of ME, viscosity, and transparency of LC and turbidity of EM was observed. The preliminary formulations were visually observed for clarity, phase transition, and viscosity. The appropriate formulation components were selected for formulation development $[12,13]$. 


\subsubsection{Preparation of experimental batches.}

Based on results of solubility, compatibility, and preliminary studies, the selected formulation components, i.e., Ethyl oleate, Cremophore EL, and Span 20, were subjected to optimization studies by constructing pseudo ternary phase diagram with the objective of optimization of the surfactant:co-surfactant (S:CoS) ratio. W/O ME Formulation batches (F1 - F5) were prepared with different $S: C o S$ ratio $(1: 1,1: 2,1: 3,2: 1,3: 1)$. The various $S: C o S$ ratios at constant oil was titrated with an aqueous phase. The formulations were prepared with the same method as described in section 2.2.2 with varying $\mathrm{S}: \mathrm{CoS}$ ratios [8]. The optimization was conducted by constructing the pseudo ternary phase diagram. Table 2 outlines the composition of the tested formulations.

Table 2. Composition of Experimental Batches.

\begin{tabular}{l|c|c|c|c|c}
\multirow{2}{*}{ Ingredients } & \multicolumn{5}{|c}{ Quantity(For 100ml) } \\
\cline { 2 - 6 } & F1 & F2 & F3 & F4 & F5 \\
\hline Timolol maleate eq. to Timolol* & $680 \mathrm{mg}$ & $680 \mathrm{mg}$ & $680 \mathrm{mg}$ & $680 \mathrm{mg}$ & $680 \mathrm{mg}$ \\
\hline Ethyl oleate & $40 \mathrm{ml}$ & $40 \mathrm{ml}$ & $40 \mathrm{ml}$ & $40 \mathrm{ml}$ & $40 \mathrm{ml}$ \\
\hline Cremophore EL & $27.50 \mathrm{ml}$ & $18.34 \mathrm{ml}$ & $13.75 \mathrm{ml}$ & $36.66 \mathrm{ml}$ & $41.25 \mathrm{ml}$ \\
\hline Span 20 & $27.50 \mathrm{ml}$ & $36.66 \mathrm{ml}$ & $41.25 \mathrm{ml}$ & $18.34 \mathrm{ml}$ & $13.75 \mathrm{ml}$ \\
\hline Sorbic acid & $0.1 \mathrm{ml}$ & $0.1 \mathrm{ml}$ & $0.1 \mathrm{ml}$ & $0.1 \mathrm{ml}$ & $0.1 \mathrm{ml}$ \\
\hline Artificial Tear fluid & $5 \mathrm{ml}$ & $5 \mathrm{ml}$ & $5 \mathrm{ml}$ & $5 \mathrm{ml}$ & $5 \mathrm{ml}$ \\
\hline
\end{tabular}

$* 6.8 \mathrm{mg}$ of Timolol maleate USP is equivalent to $5 \mathrm{mg}$ of Timolol

\subsection{Characterization of experimental batches.}

2.3.1. Physical characteristics of the formulation.

The clarity during phase transition was measured by estimating \% transmittance (\%T) against distilled water by the UV Visible spectrophotometer at $650 \mathrm{~nm}$ wavelength. Conductivity during phase transition of the prepared batches was measured by calibrated conductometer. The $\mathrm{pH}$ of the microemulsion was measured using a calibrated $\mathrm{pH}$ meter[11]. The rheological characteristic of the formulation batches during phase transition was determined by Brookfield viscometer with CP-40 spindle at room temperature [18].

\subsubsection{Drug content.}

The drug content was determined by taking $1 \mathrm{ml}$ of the formulation sample and added into $50 \mathrm{ml}$ of volumetric flask. The sample was diluted with diluent medium (water and acetonitrile in the ratio of 60:40) up to $50 \mathrm{ml}$ followed by sonication for about 15-20 minutes and analyzed for Timolol maleate concentration using optimized HPLC conditions against working standard area $[19,20]$.

\subsubsection{In-vitro drug release study.}

Drug release from all the three phases during phase transition was studied using Franz diffusion cell. The formulation was loaded in the donor compartment and artificial tear fluid in the receptor compartment of the Franz diffusion cell. A treated cellophane membrane was placed between them. The assembly was stirred at $150 \mathrm{RPM}$ and $32 \pm 1^{\circ} \mathrm{C}$ to mimic the in-vivo condition. The aliquots from the receptor compartment were taken periodically and replaced with fresh ATF $[19,20]$. The samples were analyzed for drug content by HPLC, as described in the drug content section. 


\subsubsection{In-vitro gelling capacity test.}

One $\mathrm{ml}$ of optimized formulation was added to a vial containing $2 \mathrm{ml}$ of ATF kept at $37 \pm 1^{\circ} \mathrm{C}$ temperature. As the formulation comes in contact with ATF, it converts into a stiff gel, which was observed and graded according to its stiffness [21].

2.3.5. Globule size, Zeta potential, and Poly dispersibility index determination.

Globule size, globule size distribution, and zeta potential of microemulsion were determined using Zetatrac by filling the sample in an insulating sample cell. Zetatrac determines Zeta potential by measuring the response of charged particles to an electric field. Globule size distribution is determined from the velocity distribution of particles suspended in a dispersing medium, using the principle of dynamic light scattering [8].

Poly dispersibility or heterogeneity index is a measure of the distribution of molecular mass in a given sample. It determines the size range of particles in the system. The value should be less than or equal to 0.3 . It is expressed in terms of poly dispersibility index (PDI), which is measured by equation 1 .

$$
P D I=\frac{\text { Numberofglobuleshavingparticlesize }>100 \mathrm{~nm}}{\text { Numberof globuleshavingparticlesize }<100 \mathrm{~nm}} \quad \text { Equation (1) }
$$

\subsection{Characterization of the optimized formulation.}

\subsubsection{Ocular pharmacodynamic study.}

Rabbits (New Zealand white, Male, 2.5 to $3.2 \mathrm{~kg}$ ) were used for the comparative study of both optimized and marketed formulations. Animals were treated as prescribed in the NIH publication "Guide for the Care and Use of Laboratory Animals". All experiments conformed to the ARVO Resolution on the Use of Animals in Research. They were carried out under veterinary supervision, and the protocols were approved by the Ethical-Scientific Committee of the University. The animals were housed individually in standard cages in a room with normal controlled lighting, at normal room temperature $\left(16-22^{\circ} \mathrm{C}\right)$ and humidity $(30-70 \%$ relative humidity), with no restriction of food or water. During the experiments, the rabbits were placed in restraining boxes to which they had been habituated, in a room with dim lighting; they were allowed to move their heads freely, and their eye movements were not restricted.

Rabbits were divided into two groups $(n=3)$ based on body weights. The optimized formulation was instilled in the left eye of group 1 rabbits, whereas the commercially available formulation was instilled in the left eye of group 2 rabbits. In all rabbits, the right eye was instilled with a placebo in the form of a vehicle. The dosing was provided with an eyedropper (35-50 $\mu \mathrm{L})$. During the study of formulation, the rabbit eyes were assessed every day for tearing, discharge, blepharospasm (twitchy and forceful blinking of the eyelids), ptosis (eyelid drooping), and conjunctival redness, which are all signs of ocular discomfort. The assessment was carried as mentioned in OECD (Organization for Economic Co-operation and Development [OECD, 1987]) guidelines. At a predetermined time period, the IOP measurements were performed using a tonometer (TONOVET, Finland). The measurement was done in triplicate [22-25]. 


\subsubsection{Accelerated stability study.}

Accelerated stability study was conducted on optimized formulation according to ICH (International Conference on Harmonization) guidelines. An optimized formulation in its final primary packaging container was kept in stability chambers at $40^{\circ} \mathrm{C} \pm 2^{\circ} \mathrm{C} /$ not more than (NMT) $25 \% \mathrm{RH}$. The samples were withdrawn at 0,3 , and 6 months interval and were analyzed for drug content, $\mathrm{pH}$, in-vitro drug release, viscosity, $\%$ transmittance, globule size, zeta potential, and in-vitro gelling capacity [26].

\section{Results and Discussion}

\subsection{Screening of formulation components.}

The results of the solubility study are described in Figure 1. Based on the results of solubility studies, it can be seen that the Timolol maleate shows better solubility in ethyl oleate, Cremophore EL, and span 20 as compare to Isopropyl myristate, Capmul MCM, Tween 20, Tween 80, and Solutol HS. The results of one-month drug-excipient compatibility studies revealed compatibility of Timolol maleate with all the selected formulation components except Capmul MCM where phase separation of drug with Capmul MCM was observed, which can be due to lower solubility of the drug in the oil. The results are shown in Table 3.

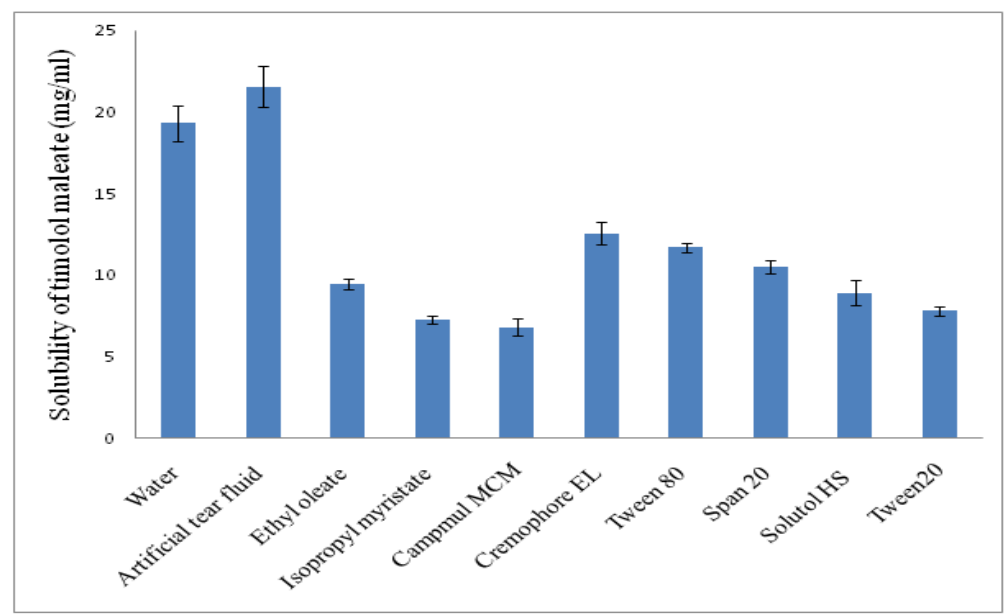

Figure 1. Solubility study of Timolol maleate in a different solvent.

Table 3. Compatibility of Timolol maleate with formulation excipients.

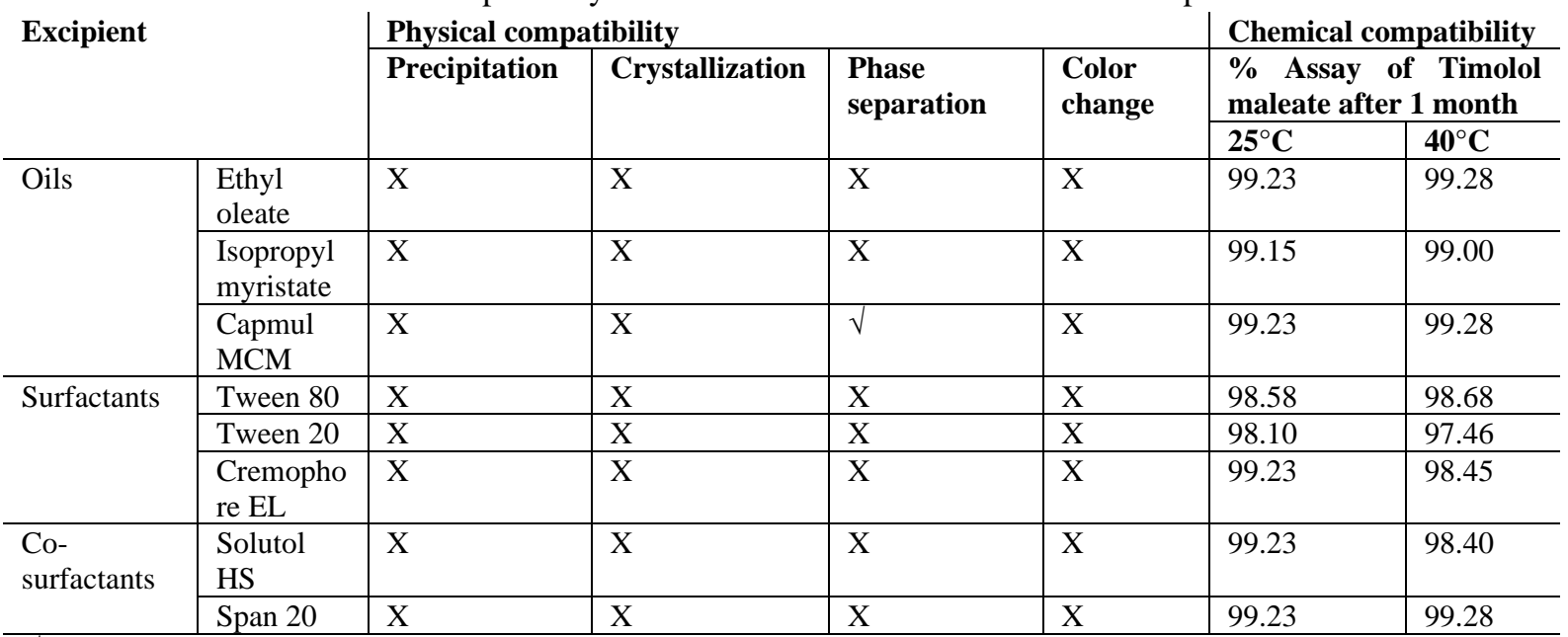

$\sqrt{ }=$ Present; $X=$ Absent 


\subsection{Preliminary batches.}

All the batches (A to $\mathrm{H}$ ) showed phase transition from w/o ME to o/w ME with LC phase as intermediate phase upon dilution. The general observation shows that all preliminary batches remained in w/o ME state for up to nearly $25 \%$ of water content. As the water content increases beyond that upon dilution, the system converts into LC state. When the water content reaches beyond $75 \%$, the phase inversion takes place to $\mathrm{o} / \mathrm{w}$ coarse emulsion. The general trend of the above behavior is shown in Figure 2. The phase diagram shows both $\mathrm{CE}$ and LC region above ME region as prospective phase changes. ME made from nonionic surfactants are sensitive to dilution changes due to changes in the affinity of surfactant in water or oil, which governs interfacial curvature. With an increase in aqueous content, the solubility of surfactant molecules may change from oil soluble to water-soluble, leading to the formation of o/w $\mathrm{CE}$ with an intermediate LC phase. This dilution of composition takes place in-vivo with tear fluid in physiological conditions.

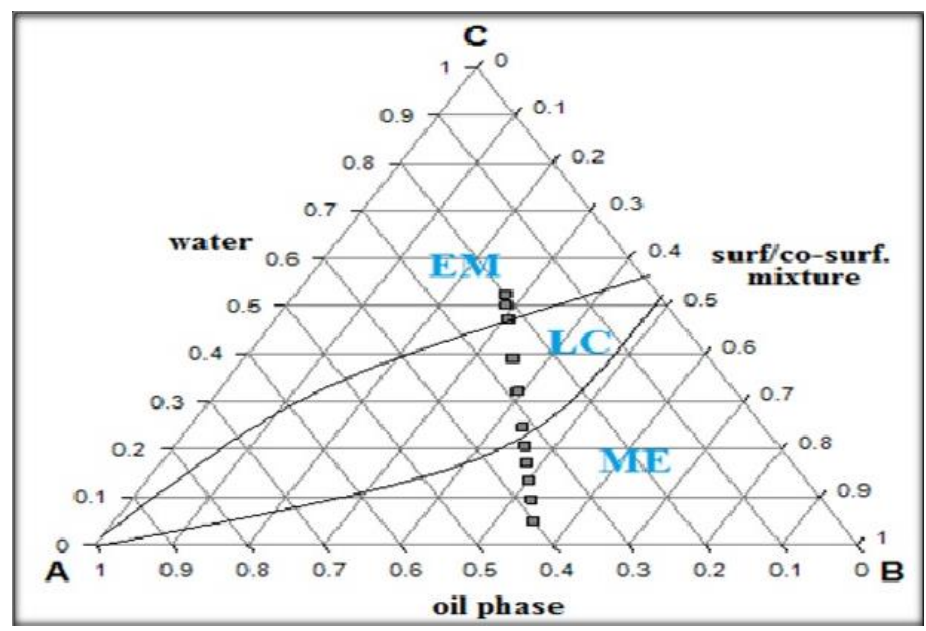

Figure 2. Pseudo ternary phase diagram for Preliminary batches.

Visual observation was used to identify LC, ME, and CE phases. LC transition was identified by semisolid appearance while $\mathrm{CE}$ was identified by the turbid less viscous transition. The primary batches A to G shows less transparency and viscosity during LC phase transition compared to batch $\mathrm{H}$. This may be due to comparatively lesser solubility of Isopropyl myristate, tween 80, and Solutol HS. Ethyl oleate, Cremophore EL, and Span 20 appeared as better components for the transparent LC phase with acceptable viscosity, which serves the objective of this study. Hence components of batch H, i.e., Ethyl oleate as oil, Cremophore EL as a surfactant, and Span 20 as co-surfactant, were selected for formulation development.

The choice of oil and surfactant is critical for the ME formulation. From the preliminary studies, it was observed that the phase transition depends on the S:CoS ratio. Cremophore EL has emerged as a good surfactant and solubilizer with good ophthalmic tolerance. Span 20 is a nonionic ester of sorbitan oleate and is approved by FDA for ophthalmic use. Ethyl oleate as oil shows good solubility and compatibility with the drug. Thus Ethyl oleate, Cremophore EL, and Span 20 were selected as oil, surfactant, and co-surfactant, respectively.

\subsection{Preparation of experimental batches.}

The formulation batches were prepared to keep in mind the objective of this study to find an optimized composition with the potential of LC phase transition. The pseudo ternary 
systems were prepared using the titration method based on selected components. The pseudo ternary phase diagram of five different compositions prepared using five different $\mathrm{S}: \mathrm{CoS}$ ratios are shown in Figure 3. Here ME region is showed red color while LC region is shown in blue color. It is evident from the figure that the formulation F1 and F4 with the S:CoS ratio 1:1 and 2: 1 respectively show a larger ME region (red) compared to the rest of the ratios and eventually form transparent LC gel formation upon dilution. All the formulations (F1-F5) were subjected to further evaluation to find the final optimized formulation.
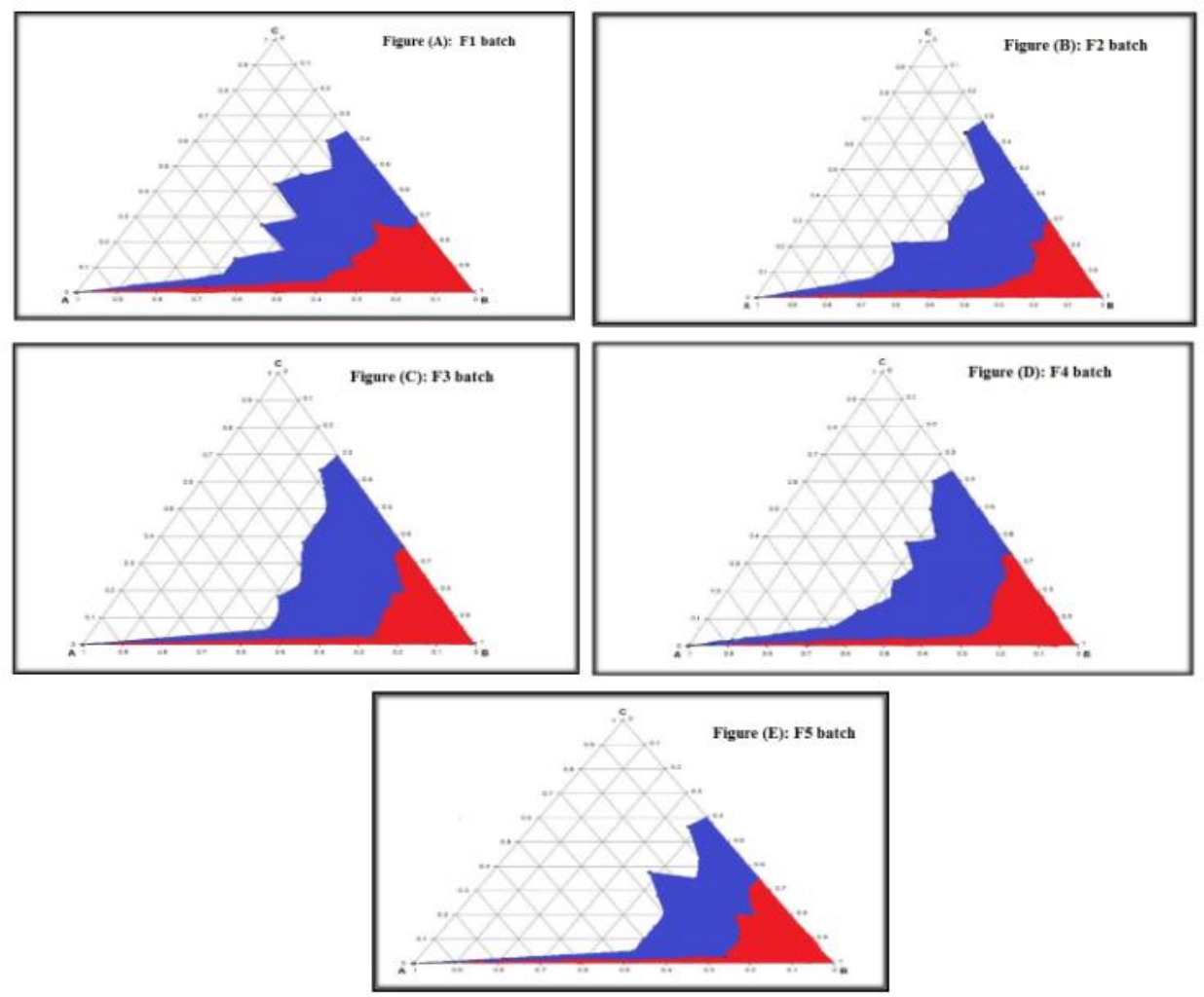

Figure 3. Pseudo ternary phase diagram for Formulation batches F1-F5.

3.4. Characterization of Experimental Batches.

3.4.1. Clarity, $\mathrm{pH}$, and drug content.

The clarity of w/o ME formulations and their LC in-situ gel state was measured and depicted in Table 4. Formulations F1 and F4 showed more than 98\%transmittance in w/oME state, which indicates a comparatively smaller globule size compared to other batches. The LC phase also showed comparatively better clarity for F1 and F4 formulations. The picture of clarity of formulation F4 in ME and LC state is shown in Figure 4.

Table 1. Clarity, $\mathrm{pH}$, drug content, and in-vitro gelling study results.

\begin{tabular}{l|l|l|l|l|l|l|l}
\multirow{2}{*}{ Batch } & \multirow{2}{*}{ Ratio } & \multicolumn{2}{|l|}{ \% T before and after phase transition } & $\mathbf{p H}$ & $\begin{array}{l}\text { \% Timolol } \\
\text { maleate content }\end{array}$ & $\begin{array}{l}\text { In-vitro } \\
\text { gelling study }\end{array}$ \\
\cline { 3 - 4 } & & w/o ME & LC in situ gel state & & 6.7 & $98.76 \pm 0.075$ & +++ \\
\hline F1 & $(1: 1)$ & $98.1 \pm 0.18$ & $86.2 \pm 0.32$ & 6.5 & $97.37 \pm 0.004$ & ++ \\
\hline F2 & $(1: 2)$ & $94.8 \pm 0.71$ & $69.7 \pm 0.11$ & 7.3 & $92.51 \pm 0.025$ & +++ \\
\hline F3 & $(1: 3)$ & $92.4 \pm 0.54$ & $73.0 \pm 0.43$ & 7.2 & $99.64 \pm 0.003$ & ++++ \\
\hline F4 & $(2: 1)$ & $99.3 \pm 0.21$ & $89.3 \pm 0.22$ & 7.5 & $95.27 \pm 0.061$ & ++ \\
\hline F5 & $(3: 1)$ & $89.5 \pm 0.35$ & $71.7 \pm 1.15$ &
\end{tabular}

$+=$ Gelation after few min and remain for few hours, $++=$ Gelation immediate and few for hours, $+++=$ Gelation immediate and remain extended time, $++++=$ Very high viscosity 
Inferior clarity of the remaining w/o ME formulations indicates a higher globule size and result in a translucent LC state. The $\mathrm{pH}$ of the formulations was found to be in the range of 6.7 to 7.5 for all formulations and hence compatible with the physiological requirement.
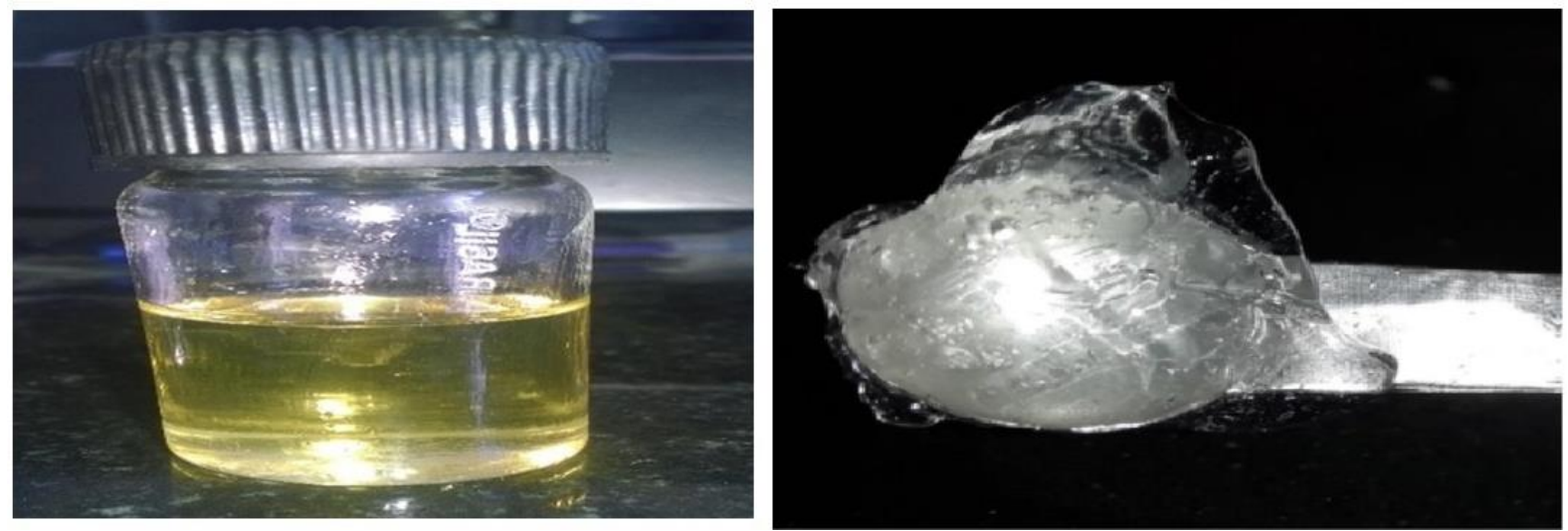

Figure 4. The physical appearance of w/o microemulsion and LC in-situ gelling.

\subsubsection{Rheological study.}

The previous literature and our studies revealed an increase in viscosity with an increase in water content, minimum in w/o ME state to reaching its maximum with LC state and dropping back to a minimum with subsequent phase transition to EM. The objective of creating LC phase is to increase in-situ viscosity to for enhancing longer ocular retention. The rheological behavior of the formulations under investigation is reported in Figure 5.

The w/o ME showed Newtonian flow with low viscosity. As the phase transition takes place from ME to LC, the flow becomes pseudoplastic. The sudden change in viscosity is explained by the formation of the lamellar LC system due to the interactions between the comprising surfactant molecules.

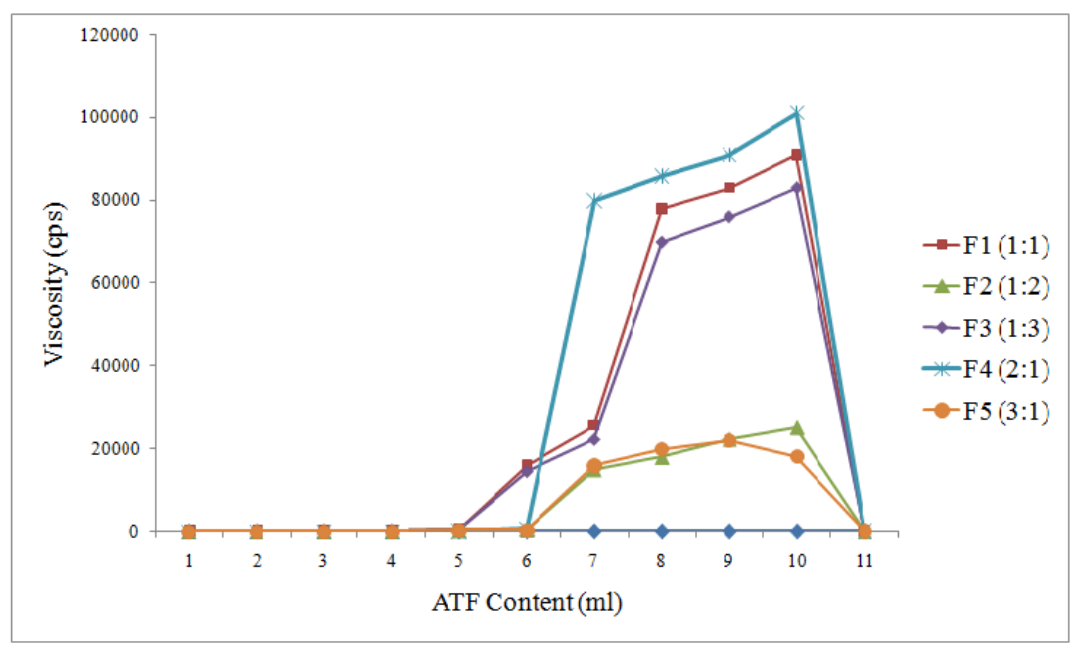

Figure 5. Rheological behavior of experimental batches.

Due to the pseudoplastic nature of LC state, upon increasing the rate of shear, the LC structure becomes perturbed, making the intermolecular attraction weak. This conversion into shear-thinning flow behavior is favorable for ocular topical drug delivery, where the viscosity reduces upon blinking of the eyelid. Further dilution with tear fluid leads to breaking of LC structure followed by the formation of $\mathrm{o} / \mathrm{w}$ EM. This phase transition is indicated by a sharp decrease in viscosity and conversion to Newtonian flow. 
Formulation F4 shows the highest viscosity during the LC state while the viscosity of remaining formulations found in descending order as $\mathrm{F} 4>\mathrm{F} 1>\mathrm{F} 3>\mathrm{F} 2>\mathrm{F} 5$. The results show the effect of S:CoS ratio on rheological behavior.

\subsubsection{Conductance measurement.}

Conductivity measurement showed almost zero values for formulations before phase transition in ME state, indicating oil as the external phase. As the water content increases during phase transition to LC state and beyond, the drastic increase in conductivity was observed, indicating the formation of an o/w EM system. The results of the conductivity study demonstrating the phase transition are depicted in Figure 6.

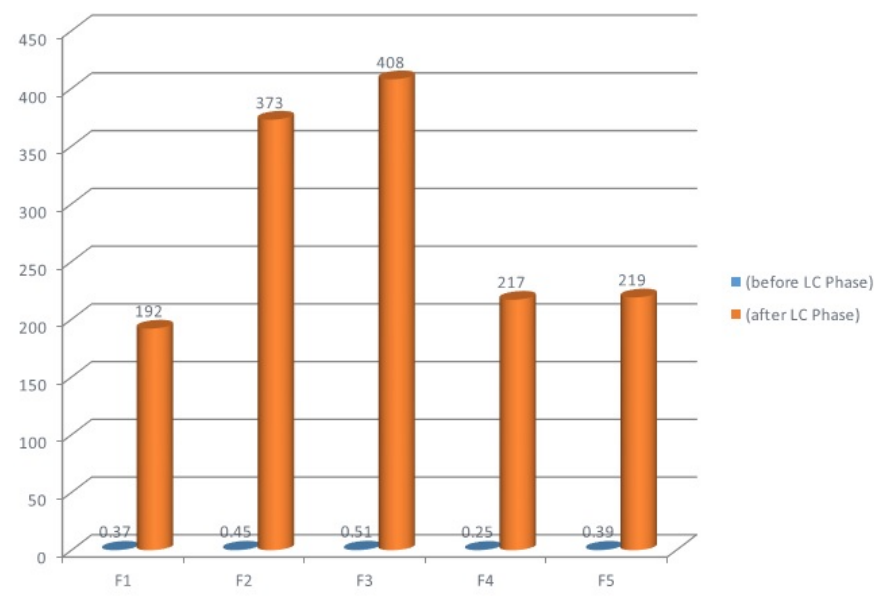

Figure 6. Conductance of formulation batches.

\subsubsection{In-vitro drug release study.}

Figure 7 and Figure 8 show the in-vitro release profile of Timolol maleate form the w/o ME and LC state, respectively. The cumulative amount of Timolol that had permeated through the membrane (\%) was plotted as a function of time (hours). Similar to the rheological study, the drug release also depended on the water content of the formulations. During both w/o ME state and LC state, the water content is less compared to EM state.

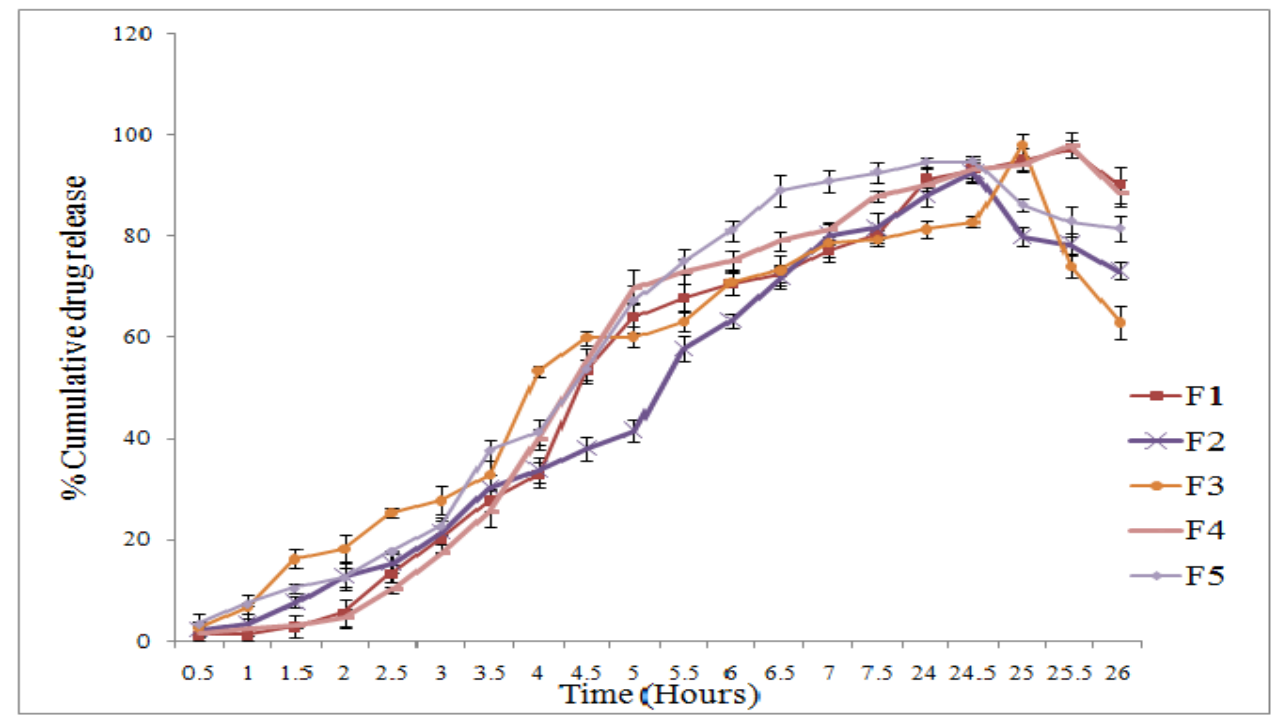

Figure 7. Drug release study of w/o microemulsion. 


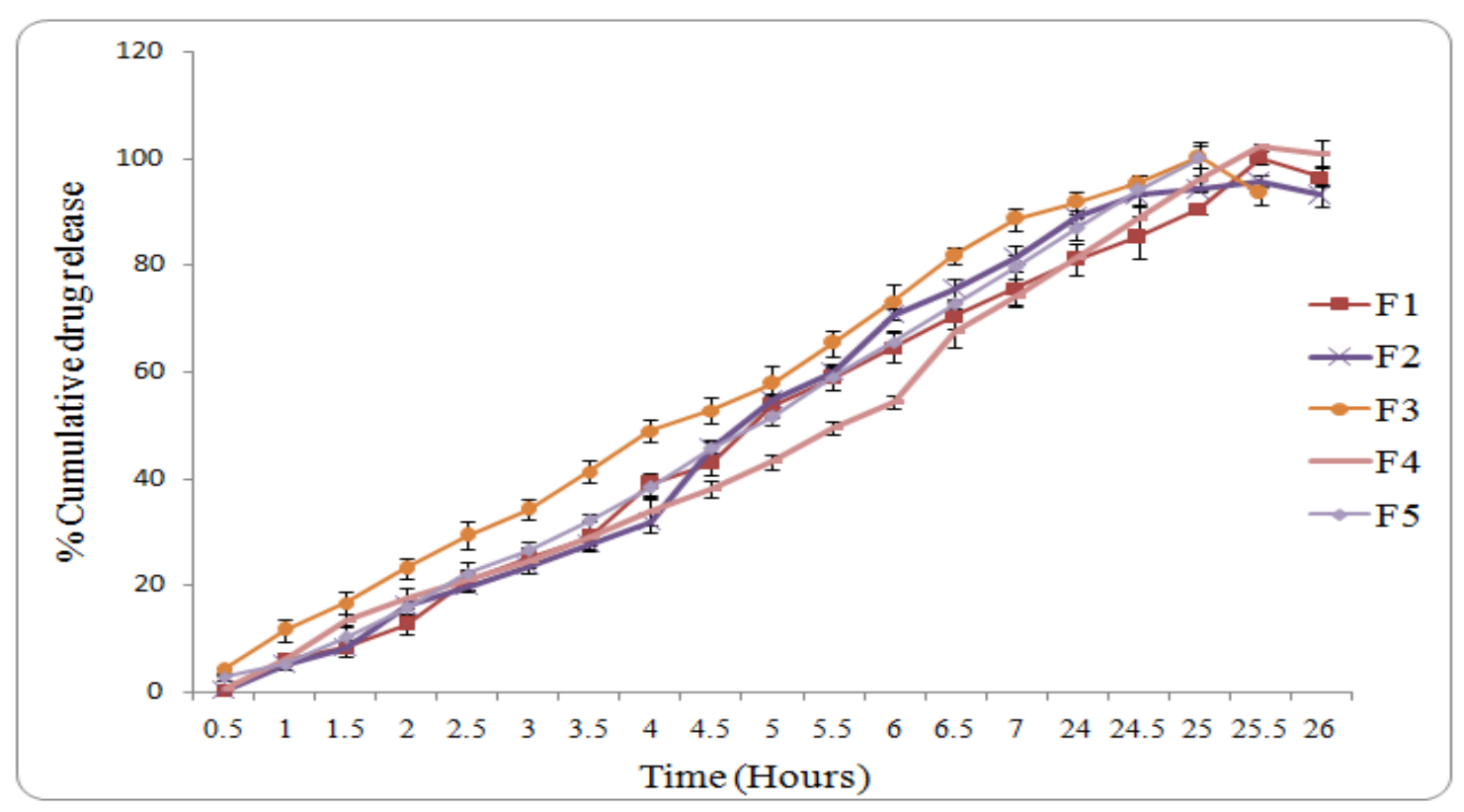

Figure 8. Drug release of liquid crystalline phase.

The result revealed a controlled \% drug release for up to 24 hours from w/o ME and LC state suggesting the ability of the formulation to decrease dosing frequency. The drug release profile of optimized formulation shows linear drug release with fewer fluctuations in $\%$ drug release.

\subsubsection{In-vitro gelling capacity test.}

Gelling capacity is an important requirement of LC state. The optimum gelling of the formulation allows easy administration and rapid gelling at the physiological condition. The gelling capacity of optimized formulation was evaluated on the basis of flowability and visual evaluation of gel stiffness and its retention time. We assessed the gel capacity on a grading scale between - and ++++ . The grades of gelation were recorded as: (-) No gelation, $(+)$ weak gelation remains up to $10 \mathrm{~min},(++)$ Immediate gelation remains for up to $5 \mathrm{hrs}$ (less stiff gel), (+ ++ ) Immediate gelation remains for longer period up to $10 \mathrm{hrs}$ (stiff gel), $(++++)$ Immediate gelation remains for extended period for more than $12 \mathrm{hrs}$ (very stiff gel).

The results shown in Table 4 indicate the in-vitro gelling capacity of the experimental formulations by means of visual gelling observation. During the physiological condition, all formulations showed immediate gelation within a period of 5-10 seconds. This short gelation time indicates that the formulation will not get drained due to eyelid blinking.

\subsubsection{Globule size, polydispersity index, zeta potential.}

Based on the results obtained from the above studies, formulation F1 and F4 were shortlisted for globule size determination considering their greater clarity, rheology, and invitro gelling capacity. Both the formulations F1 and F4 were subjected to droplets size measurement. The mean globule size of both formulations is shown in Table 5. The globule size is found to be in the desired size range $(10-200 \mathrm{~nm})$ in the case of both the formulations indicating the potential of good permeation through the biological membrane. The PDI value 
of both the formulations was found to be less than 0.3, indicating uniform globule size distribution.

The zeta potential values of formulation $\mathrm{F} 1$ were found out to be $-13.75 \mathrm{mV}$ while that of formulation $\mathrm{F} 4$ was found out to be $17.9 \mathrm{mV}$. The zeta potential values suggest physical stability on storage.

Table 5. Globule size, Zeta potential, and Polydispersity index of F1 \& F4 batches.

\begin{tabular}{l|c|c|c|c}
\multicolumn{5}{c}{ For w/o Microemulsion } \\
\hline Batch no & Ratio & Globule size & PDI & Zeta potential \\
\hline F1 & $(1: 1)$ & $23.47 \mathrm{~nm}$ & 0.253 & $-13.75 \mathrm{mV}$ \\
\hline F4 & $(2: 1)$ & $19.61 \mathrm{~nm}$ & 0.27 & $17.9 \mathrm{mV}$ \\
\hline \multicolumn{5}{c}{ For Liquid Crystalline Phase } \\
\hline F1 & $(1: 1)$ & $40.70 \mathrm{~nm}$ & 0.14 & $13.2 \mathrm{mV}$ \\
\hline F4 & $(2: 1)$ & $36.40 \mathrm{~nm}$ & 0.245 & $-21.5 \mathrm{mV}$ \\
\hline \multicolumn{5}{c}{ For Coarse emulsion o/w } \\
\hline F1 & $(1: 1)$ & $6000 \mathrm{~nm}$ & 0.127 & $24.4 \mathrm{mV}$ \\
\hline F4 & $(2: 1)$ & $2317 \mathrm{~nm}$ & 0.201 & $-20.52 \mathrm{mV}$
\end{tabular}

\subsection{Characterization of Optimized Batch.}

Based on the results obtained from clarity, viscosity, in-vitro drug release, globule size, and zeta potential measurement formulation F4 with $\mathrm{S}: \mathrm{CoS}$ ratio of 2:1 was selected as the optimized formulation. The composition of the optimized batch is shown in Table 6, with its characterization data depicted in Table 7. The drug release profile of optimized formulation shows linear drug release, as shown in Figure 9. The drug release profile after sol to gel transformation of in situ gelling showed linearity with the square root of time and followed Higuchi's equation. The drug release was found similar to the marketed formulation with fewer fluctuations in \% drug release.

Table 6. Composition of optimized batch.

\begin{tabular}{l|l|l} 
Sr. No. & Ingredients & $\begin{array}{l}\text { Quantity } \\
\text { (For 100ml) }\end{array}$ \\
\hline 1 & Timolol maleate eq. to Timolol* & $680 \mathrm{mg}$ \\
\hline 2 & Ethyl oleate & $40 \mathrm{ml}$ \\
\hline 3 & Cremophore EL & $36.66 \mathrm{ml}$ \\
\hline 4 & Span 20 & $18.34 \mathrm{ml}$ \\
\hline 5 & Sorbic acid & $0.1 \mathrm{ml}$ \\
\hline 6 & Artificial Tear fluid & $5 \mathrm{ml}$ \\
\hline$* 6.8 \mathrm{mg}$ of Timolol maleate USP is equivalent to $5 \mathrm{mg}$ of Timolol
\end{tabular}

Table 7. Evaluation of optimized batch.

\begin{tabular}{l|l|l} 
Sr. No. & Parameter & Results \\
\hline 1. & \% Transmittance & $99.3 \pm 0.21$ \\
\hline 2. & Viscosity(at 100 RPM) & $105.01 \mathrm{cps} \pm 10 \mathrm{cps}$ \\
\hline 3. & \% cumulative drug release & $98.4 \pm 2.45$ \\
\hline 4. & Assay & $99.64 \pm 0.003$ \\
\hline 5. & Conductance & $0.25 \pm 0.053$ \\
\hline 6. & pH & $7.2 \pm 0.2$ \\
\hline 7. & Globule size & $19.61 \mathrm{~nm} \pm 2 \mathrm{~nm}$ \\
\hline 8. & PDI & 0.37 \\
\hline 9. & Zeta potential & $17.9 \mathrm{mV} \pm 0.3 \mathrm{mV}$ \\
\hline 10. & In-vitro gelling capacity & $\begin{array}{l}\text { Gelation immediate and remained for an extended } \\
\text { time period }\end{array}$
\end{tabular}




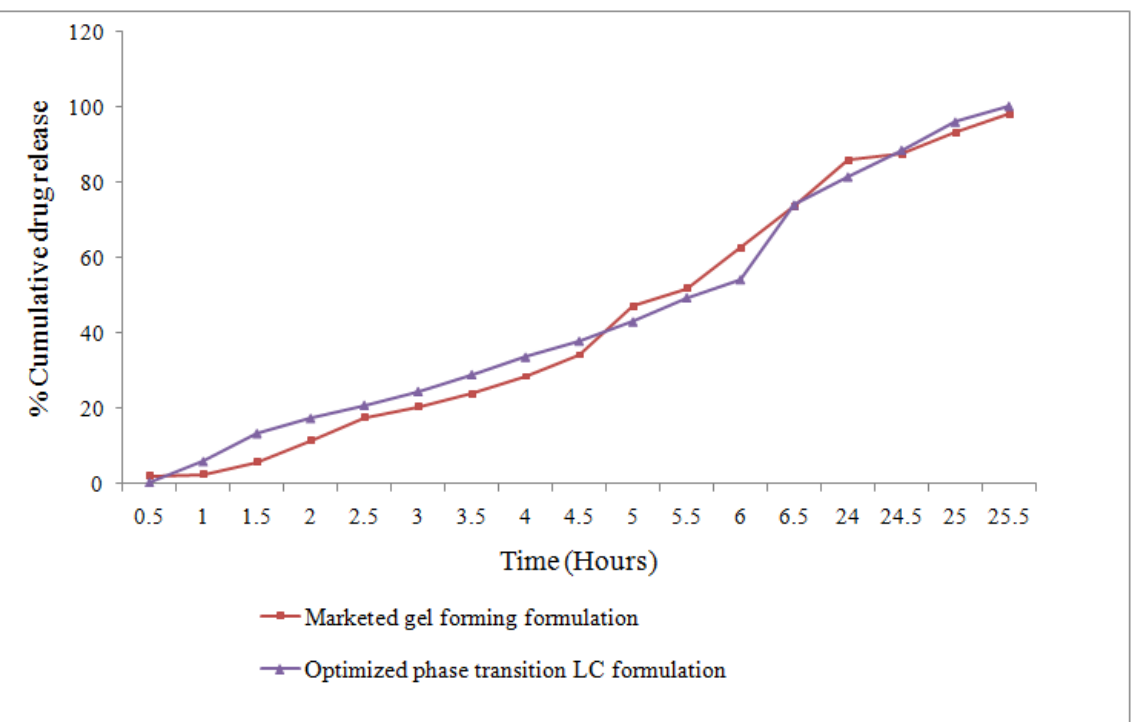

Figure 9. Comparative in-vitro drug release study (Market formulation vs. Optimized formulation).

\subsubsection{Pharmacodynamic study.}

The in vivo pharmacodynamic study was carried out in an experimental model using 2 groups of normotensive Rabbits. The normal baseline for Intraocular pressure (IOP) was observed at $15.05 \mathrm{mmHg}$. No significant day to day variation $(\mathrm{p}=0.423)$ was observed in the normal IOP measurement for each animal. There was no significant difference $(p=0.348)$ detected in both groups. In this study left eye of group 1 treated with marketed gel-forming preparation (1 drop), and Group 2 left eye treated with optimized phase inversion w/o ME (1 drop $=40$ to $50 \mu \mathrm{L}$ ) while the vehicle is treated in all group animals in the right eye to make a baseline for study. The IOP reduction in both treated groups was found similar, as showed in Figure 10. To eliminate fluctuations due to diurnal IOP variations, the IOP values were expressed as the difference from the corresponding baseline values. The results suggest the improvement in drug residence time of Timolol based phase inversion w/o ME, which will reduce the therapeutic dosage of drugs. In vitro, drug release profile showed 25 hours therapeutic release, while the in-vivo study showed sustained therapeutic effect (reduction in IOP), which suggests the potential of microemulsion for sustained drug delivery.

As described in the drug release study earlier, the in-vitro drug release profile showed sustained drug release, which is reassured by the in-vivo study, which showed a sustained therapeutic effect (reduction in IOP). The results suggest the potential of optimized formulation for sustained drug delivery. An IOP reduction study indicates that optimized formulation was equally efficacious with less variability in the reduction of IOP among the subjects when compared to marketed formulation. It also demonstrates that once-daily dosing is enough for the optimized formulation of Timolol maleate for ophthalmic delivery.

During in vivo pharmacodynamic study in rabbits, there were observed eyelids, conjunctiva; cornea was observed visually. The result of this test showed no opacity, conjunctival chemosis, redness, discharge, or no iris alteration observed in any of the rabbits after observation of the Rabbits eyes; it would appear that the phase transition w/o ME formulation is non-irritating. 


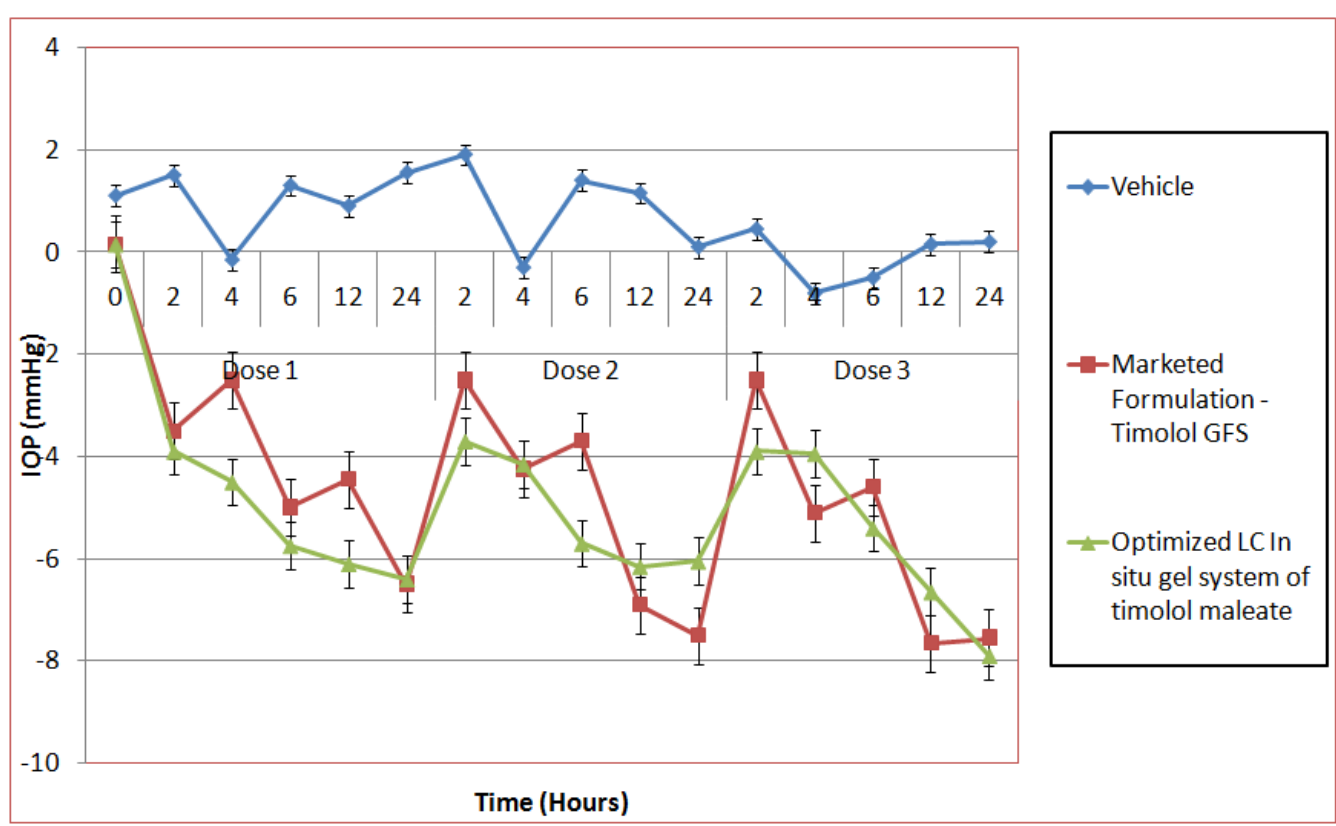

Figure 10. In-vivo pharmacodynamic study results in rabbits.

\subsubsection{Accelerated stability study.}

Accelerated stability study data revealed that the formulation remained stable over a period of 6 months at elevated conditions. As shown in Table 8, there is no significant change in the $\mathrm{pH}$ and the assay of the formulation indicating the chemical stability of the formulation. The microemulsion is physically stable, as evidenced by the visual observation, and absence of signs of instability such as phase separation. Also, there is a negligible change in the globule size of the system stored at the elevated conditions. After 6 month interval, change in the zeta potential was found to be non-significant. All these results suggest that the formulation F4 is physically and chemically stable on storage.

Table 8. Accelerated stability study results of optimized batch.

\begin{tabular}{|c|c|c|c|c|}
\hline \multirow{2}{*}{ Sr. No } & \multirow{2}{*}{ Testing parameters } & \multicolumn{3}{|c|}{ Storage period at $40 \pm 2^{\circ} \mathrm{C}$ temperature and NMT $25 \%$ RH } \\
\hline & & 0 Month & 3 Months & 6 Months \\
\hline 1 & Appearance & Clear & Clear & Clear \\
\hline 2 & Clarity $(\%)$ & 98.8 & 98.1 & 97.9 \\
\hline 3 & Viscosity (cps) & 96 & 90 & 94 \\
\hline 4 & Assay of Timolol maleate $(\%)$ & $99.10 \%$ & $97.70 \%$ & $96.73 \%$ \\
\hline \multirow{8}{*}{5} & \multicolumn{4}{|l|}{ Related substances } \\
\hline & Timolol related compound B $(\%)$ & 0.018 & 0.201 & 0.305 \\
\hline & Timolol related compound D (\%) & 0.980 & 1.180 & 1.540 \\
\hline & Timolol related compound E (\%) & Not Detected & Not Detected & Not Detected \\
\hline & Timolol related compound C (\%) & 0.147 & 0.204 & 0.501 \\
\hline & Timolol related compound F (\%) & 0.490 & 0.570 & 0.910 \\
\hline & Any highest unspecified impurity (\%) & 0.054 & 0.087 & 0.150 \\
\hline & Total degradation products $(\%)$ & 1.689 & 2.242 & 3.406 \\
\hline 6 & Conductance $(\mathrm{mS})$ & $0.24 \pm 0.55$ & $0.27 \pm 1.01$ & $0.29 \pm 0.47$ \\
\hline 7 & Globule size $(\mathrm{nm})$ & 19.61 & - & 20.73 \\
\hline 8 & PDI & 0.37 & - & 0.394 \\
\hline 9 & Zeta potential $(\mathrm{mV})$ & -17.9 & - & -20.5 \\
\hline 10 & Osmolality (mOsm/kg) & 312 & 308 & 315 \\
\hline 11 & In-vitro gelling capacity & +++ & +++ & +++ \\
\hline 12 & In-vitro drug release (at $24 \mathrm{Hr}$ ) & $97.90 \%$ & $96.34 \%$ & $95.23 \%$ \\
\hline
\end{tabular}




\section{Conclusions}

Phase transition w/oME of Timolol maleate was prepared by the auto-emulsification method. This method was found to be simple, did not require specialized equipment, and scaleup feasibility. Upon administration into the eye, it will transform from w/o ME to o/w EM with intermediate-high viscosity LC state by simultaneous dilution with secreted tear fluid, which may increase ocular residence time. The optimized formulation exhibited all the desirable attributes of an ideal ME and was found to be stable and non-irritant to the eye. The in vitro drug release and IOP reduction with optimized formulation were found comparable and less fluctuating compared to marketed formulation. In-vivo study indicated that the microemulsion would be able to offer benefits, such as increased residence time, prolonged drug release, reduction in the frequency of administration, and thereby definitely improve patient compliance.

\section{Funding}

This research received no external funding.

\section{Acknowledgments}

The authors are grateful to Ms. Riya Pateland Ms.Bindu Yadav, Research Scholars, Dept. of Pharmaceutical Technology, Ramanbhai Patel College of Pharmacy, Charotar University of Science and Technology (CHARUSAT) for their writing assistance.

\section{Conflicts of Interest}

The authors declare no conflict of interest.

\section{References}

1. Maharjan, P.; Cho, K.H.; Maharjan, A.; Shin, M.C.; Moon, C.; Min, K.A. Pharmaceutical challenges and perspectives in developing ophthalmic drug formulations. Journal of Pharmaceutical Investigation 2019, 49, 215-228, https://doi.org/10.1007/s40005-018-0404-6.

2. Bachu, R.D.; Chowdhury, P.; Al-Saedi, Z.H.F.; Karla, P.K.; Boddu, S.H.S. Ocular Drug Delivery BarriersRole of Nanocarriers in the Treatment of Anterior Segment Ocular Diseases. Pharmaceutics 2018, 10, 2836, https://doi.org/10.3390/pharmaceutics10010028.

3. Gote, V.; Sikder, S.; Sicotte, J.; Pal, D. Ocular Drug Delivery: Present Innovations and Future Challenges. Journal of Pharmacology and Experimental Therapeutics 2019, 370, 602-624, https://doi.org/10.1124/jpet.119.256933.

4. Desai, A.; Shukla, M.; Maulvi, F.; Ranch, K. Ophthalmic and optic drug administration: novel approaches and challenges. InNovel Drug Delivery Technologies 2019, 12, 335-381, https://doi.org/10.22270/jddt.v8i6.2029.

5. Suri, R.; Beg, S.; Kohli, K. Target strategies for drug delivery bypassing ocular barriers. Journal of Drug Delivery Science and Technology 2020, 55, 101389-101400, https://doi.org/10.1016/j.jddst.2019.101389.

6. Wu, Y.; Liu, Y.; Li, X.; Kebebe, D.; Zhang, B.; Ren, J.; Lu, J.; Li, J.; Du, S.; Liu, Z. Research progress of in-situ gelling ophthalmic drug delivery system. Asian Journal of Pharmaceutical Sciences 2019, 14, 1-15, https://doi.org/10.1016/j.ajps.2018.04.008.

7. Tiwari, A.; Shukla, R.K. Novel ocular drug delivery systems: An overview. Journal of Chemical and Pharmaceutical research 2010, 2, 348-55.

8. Tiwari, R.; Pandey, V.; Asati, S.; Soni, V.; Jain, D. Therapeutic challenges in ocular delivery of lipid based emulsion. Egyptian Journal of Basic and Applied Sciences 2018, 5, 121-129, https://doi.org/10.1016/j.ejbas.2018.04.001.

9. Mourelatou EA.; Sarigiannis Y.; Petrou CC. Ocular Drug Delivery Nanosystems: Recent Developments and Future Challenges. Drug Delivery Nanosystems: From Bioinspiration and Biomimetics to Clinical Applications 2019, 15, 92-102. https://doi.org/10.1201/jop.2019.0135. 
10. Üstündağ Okur N.; Çağlar EŞ.; Siafaka PI. Novel Ocular Drug Delivery Systems: An Update on Microemulsions. Journal of Ocular Pharmacology and Therapeutics 2020, 36, 6-15. https://doi.org/10.1089/jop.2019.0135.

11. Verma, D.; Kaul, S.; Jain, N.; Nagaich, U. Fabrication and Characterization of Ocular Phase Transition Systems for Blepharitis. A Novel Approach. Drug Delivery Letters 2020, 10, 24-37, https://doi.org/10.2174/2210303109666190614115304.

12. Thakur, S.S.; Solloway, J.; Stikkelman, A.; Seyfoddin, A.; Rupenthal, I.D. Phase transition of a microemulsion upon addition of cyclodextrin - applications in drug delivery. Pharmaceutical Development and Technology 2018, 23, 167-175, https://doi.org/10.1080/10837450.2017.1371191.

13. Bharti, S.K.; Kesavan, K. Phase-transition W/O Microemulsions for Ocular Delivery: Evaluation of Antibacterial Activity in the Treatment of Bacterial Keratitis. Ocular Immunology and Inflammation 2017, 25, 463-474, https://doi.org/10.3109/09273948.2016.1139136.

14. Fatima, E. Role of Micro Emulsion Based In-Situ Gelling System of Fluoroquinolone for Treatment of Posterior Segment Eye Diseases (PSED). Journal of Drug Delivery and Therapeutics 2020, 10, 265-72, https://doi.org/10.22270/jddt.v10i3.4079.

15. El Maghraby GM.;ArafaMF.;Essa EA. Phase transition microemulsions as drug delivery systems. InApplications of Nanocomposite Materials in Drug Delivery 2018, 1, 787-803.

16. Li Y.; Angelova A.; Liu J.; Garamus VM.; Li N, Drechsler M.; Gong Y, Zou A. In situ phase transition of microemulsions for parenteral injection yielding lyotropic liquid crystalline carriers of the antitumor drug bufalin. Colloids and Surfaces B: Biointerfaces 2019, 173, 217-25. https://doi.org/ 10.1016/j.colsurfb.2018.09.023.

17. Gallarate, M.; Chirio, D.; Bussano, R.; Peira, E.; Battaglia, L.; Baratta, F.; Trotta, M. Development of O/W nanoemulsions for ophthalmic administration of timolol. International Journal of Pharmaceutics 2013, 440, 126-134, https://doi.org/10.1016/j.ijpharm.2012.10.015.

18. De Souza Ferreira, S.B.; Moço, T.D.; Borghi-Pangoni, F.B.; Junqueira, M.V.; Bruschi, M.L. Rheological, mucoadhesive and textural properties of thermoresponsive polymer blends for biomedical applications. Journal of the Mechanical Behavior of Biomedical Materials 2016, 55, 164-178, https://doi.org/10.1016/j.jmbbm.2015.10.026.

19. Mishra, G.P.; Tamboli, V.; Mitra, A.K. Effect of hydrophobic and hydrophilic additives on sol-gel transition and release behavior of timolol maleate from polycaprolactone-based hydrogel. Colloid and Polymer Science 2011, 289, 1553-1562, https://doi.org/10.1007/s00396-011-2476-y.

20. Ilka, R.; Mohseni, M.; Kianirad, M.; Naseripour, M.; Ashtari, K.; Mehravi, B. Nanogel-based natural polymers as smart carriers for the controlled delivery of Timolol Maleate through the cornea for glaucoma. International Journal of Biological Macromolecules 2018, 109, 955-962, https://doi.org/10.1016/j.ijbiomac.2017.11.090.

21. Sun, J.; Zhou, Z. A Novel Ocular Delivery of Brinzolamide Based On Gellan Gum: In Vitro And In Vivo Evaluation. Drug Design, Development and Therapy 2018, 12, 383-389, https://doi.org/10.2147/DDDT.S153405.

22. Destruel, P.; Zeng, N.; Brignole-Baudouin, F.; Douat, S.; Seguin, J.; Olivier, E.; Dutot, M.; Rat, P.; Dufaÿ, S.; Dufaÿ-Wojcicki, A.; Maury, M.; Mignet, N.; Boudy, V. In Situ Gelling Ophthalmic Drug Delivery System For The Optimization Of Diagnostic And Preoperative Mydriasis: In Vitro Drug Release, Cytotoxicity And Mydriasis Pharmacodynamics. Pharmaceutics 2020, 12, 360-379, https://doi.org/10.3390/pharmaceutics12040360.

23. Moosa, R.M.; Choonara, Y.E.; du Toit, L.C.; Tomar, L.K.; Tyagi, C.; Kumar, P.; Carmichael, T.R.; Pillay, $\mathrm{V}$. In vivo evaluation and in-depth pharmaceutical characterization of a rapidly dissolving solid ocular matrix for the topical delivery of timolol maleate in the rabbit eye model. International Journal of Pharmaceutics 2014, 466, 296-306, https://doi.org/10.1016/j.ijpharm.2014.02.032.

24. Dong, Y.R.; Huang, S.W.; Cui, J.Z.; Yoshitomi, T. Effects of brinzolamide on rabbit ocular blood flow in vivo and ex vivo. International journal of ophthalmology 2018, 11, 719-725, https://doi.org/10.18240/ijo.2018.05.03.

25. Mahboobian MM.; Seyfoddin A.; Aboofazeli R.; Foroutan SM.; Rupenthal ID. Brinzolamide-loaded nanoemulsions: ex vivo transcorneal permeation, cell viability and ocular irritation tests. Pharmaceutical development and technology 2019, 24, 600-606.https://doi.org/10.1080/10837450.2018.1547748.

26. Wang, S.; Chen, P.; Zhang, L.; Yang, C.; Zhai, G. Formulation and Evaluation of microemulsion-based in situ ion-sensitive gelling systems for intranasal administration of curcumin. Journal of Drug Targeting 2012, 20, 831-840, https://doi.org/10.3109/1061186x.2012.719230. 